\title{
IMPLICATIONS OF THE COSMIC BACKGROUND IMAGER POLARIZATION DATA
}

\author{
J. L. Sievers, ${ }^{1}$ C. Achermann, ${ }^{2}$ J. R. Bond,${ }^{1}$ L. Bronfman, ${ }^{3}$ R. Bustos,${ }^{2}$ C. R. Contaldi, ${ }^{1,4}$ C. Dickinson, ${ }^{5,6}$ \\ P. G. Ferreira, ${ }^{7}$ M. E. Jones, ${ }^{7}$ A. M. Lewis, ${ }^{1}$ B. S. Mason, ${ }^{8}$ J. May, ${ }^{3}$ S. T. Myers, ${ }^{9}$ \\ N. Oyarce, ${ }^{2}$ S. Padin, ${ }^{10}$ T. J. Pearson, ${ }^{5}$ M. Pospieszalski, ${ }^{11}$ A. C. S. Readhead, ${ }^{5}$ \\ R. ReEves, ${ }^{2}$ A. C. TAYlor, ${ }^{7}$ and S. TORres ${ }^{2}$ \\ Received 2005 October 12; accepted 2006 October 26
}

\begin{abstract}
We present new measurements of the power spectra of the $E$ mode of cosmic microwave background (CMB) polarization, the temperature $T$, the cross-correlation of $E$ and $T$, and upper limits on the $B$ mode from 2.5 yr of dedicated Cosmic Background Imager (CBI) observations. Both raw maps and optimal signal images in the $(u, v)$ plane and the sky plane show strong detections of the $E$ mode (11.7 $\sigma$ for the EE power spectrum overall) and no detection of the $B$ mode. The power spectra are used to constrain parameters of the flat tilted adiabatic $\Lambda$ CDM models: those determined from EE and TE bandpowers agree with those from TT, which is a powerful consistency check. There is little tolerance for shifting polarization peaks from the TT-forecast locations, as measured by the angular sound crossing scale $\theta=100 / l_{s}=1.03 \pm 0.02$ from EE and TE; compare with $1.044 \pm 0.005$ with the TT data included. The scope for extra out-of-phase peaks from subdominant isocurvature modes is also curtailed. The EE and TE measurements of CBI, DASI, and BOOMERANG are mutually consistent and, taken together rather than singly, give enhanced leverage for these tests.
\end{abstract}

Subject headings: cosmic microwave background - polarization

Online material: tar file

\section{INTRODUCTION}

Polarization of the cosmic microwave background (CMB) at the $\sim 10 \%$ level has been forecast for decades (e.g., Bond \& Efstathiou 1984), but only after a long experimental struggle was it detected and firmly established from measurements by the Degree Angular Scale Interferometer (DASI; Kovac et al. 2002), Wilkinson Microwave Anisotropy Probe (WMAP; Kogut et al. 2003), Cosmic Background Imager (CBI; Readhead et al. 2004b), CAPMAP (Barkats et al. 2005), and BOOMERANG (Masi et al. 2005; Montroy et al. 2006; Piacentini et al. 2006) experiments. The CBI (Padin et al. 2002; Readhead et al. 2004b) is a 13 element interferometer located at a height of $5080 \mathrm{~m}$ in the Chilean Andes, operating in $101 \mathrm{GHz}$ bands from $26 \mathrm{GHz}$ to $36 \mathrm{GHz}$. The CBI has been observing the polarization of the CMB since its inception in late 1999, and since 2002 September it has been operating in a polarization-optimized configuration with 42 polarizationsensitive baselines. The first CBI polarization limits (Cartwright et al. 2005) used only 12 polarization-sensitive baselines. As part of the polarization optimization, we adopted the achromatic polarizers designed by J. Kovac and described in Kovac et al. (2002). The first CBI detections in the polarization-optimized configuration included data taken from 2002 September to 2004 May

\footnotetext{
1 Canadian Institute for Theoretical Astrophysics, University of Toronto, Toronto, Canada.

2 Departamento de Ingeniería Eléctrica, Universidad de Concepción, Concepción, Chile.

3 Departamento de Astronomía, Universidad de Chile, Santiago, Chile.

4 Department of Physics, Imperial College, London, UK.

5 Chajnantor Observatory, California Institute of Technology, Pasadena, CA.

6 Jet Propulsion Laboratory, Pasadena, CA.

7 Astrophysics, Oxford University, Oxford, UK.

8 National Radio Astronomy Observatory, Green Bank, WV.

9 National Radio Astronomy Observatory, Socorro, NM.

${ }^{10}$ Kavli Institute for Cosmological Physics, Department of Astronomy and Astrophysics, University of Chicago, Chicago, IL.

11 National Radio Astronomy Observatory, Charlottesville, VA.
}

(Readhead et al. 2004b). In this paper, we present and analyze the implications of CBI polarization for our complete 2002 September2005 April polarization data set. This represents a 54\% increase in integration time over the results reported earlier (Readhead et al. 2004b).

In $\S 2$, we present an abbreviated description of the data and their analysis that leads to the compression of the data onto maps in $l$-space, a natural space for interferometry of the CMB, and further compression onto power spectra. A detailed description of the experiment, our analysis procedure, and the results of data quality tests will be given in S. T. Myers et al. (2007, in preparation). We use our improved EE and TE power spectra to test the consistency of cosmological parameters with results forecast from TT for minimal inflation-motivated tilted $\Lambda \mathrm{CDM}$ models in $\S 3.2$ and for hybrid models with an additional subdominant isocurvature component in $\S 3.4$. Special attention is paid to overall amplitudes and pattern-shifting parameters in $\S \S 3.3$ and 3.5.

\section{PROCESSING OF THE CBI POLARIZATION DATA}

\subsection{The Polarization Data and the CBI Pipeline}

The CBI instrument is described by Padin et al. (2002), and the observing and data reduction procedures used for CMB polarization studies are described in Readhead et al. (2004b). Observations were made of four mosaic fields, labeled $02^{\mathrm{h}}, 08^{\mathrm{h}}$, $14^{\mathrm{h}}$, and $20^{\mathrm{h}}$ by right ascension, each having roughly equal observing time. The $20^{\mathrm{h}}$ field was a deep $4.5^{\circ} \times 0.75^{\circ} 20^{\mathrm{h}}$ strip of six pointings separated by $45^{\prime}$. The FWHM of the CBI primary beam is $45^{\prime}$. The other three mosaics were $6 \times 6$ pointings, each covering a $4.5^{\circ} \times 4.5^{\circ}$ square. The CBI recorded visibilities on 78 baselines (antenna pairs). We note that the data in Readhead et al. (2004b) used only 12 of the 13 antennas, or 66 baselines, due to a software error, which has now been corrected. This error resulted in a $15 \%$ loss in sensitivity, but did not bias the results. The data were calibrated by reference to standard sources with an 

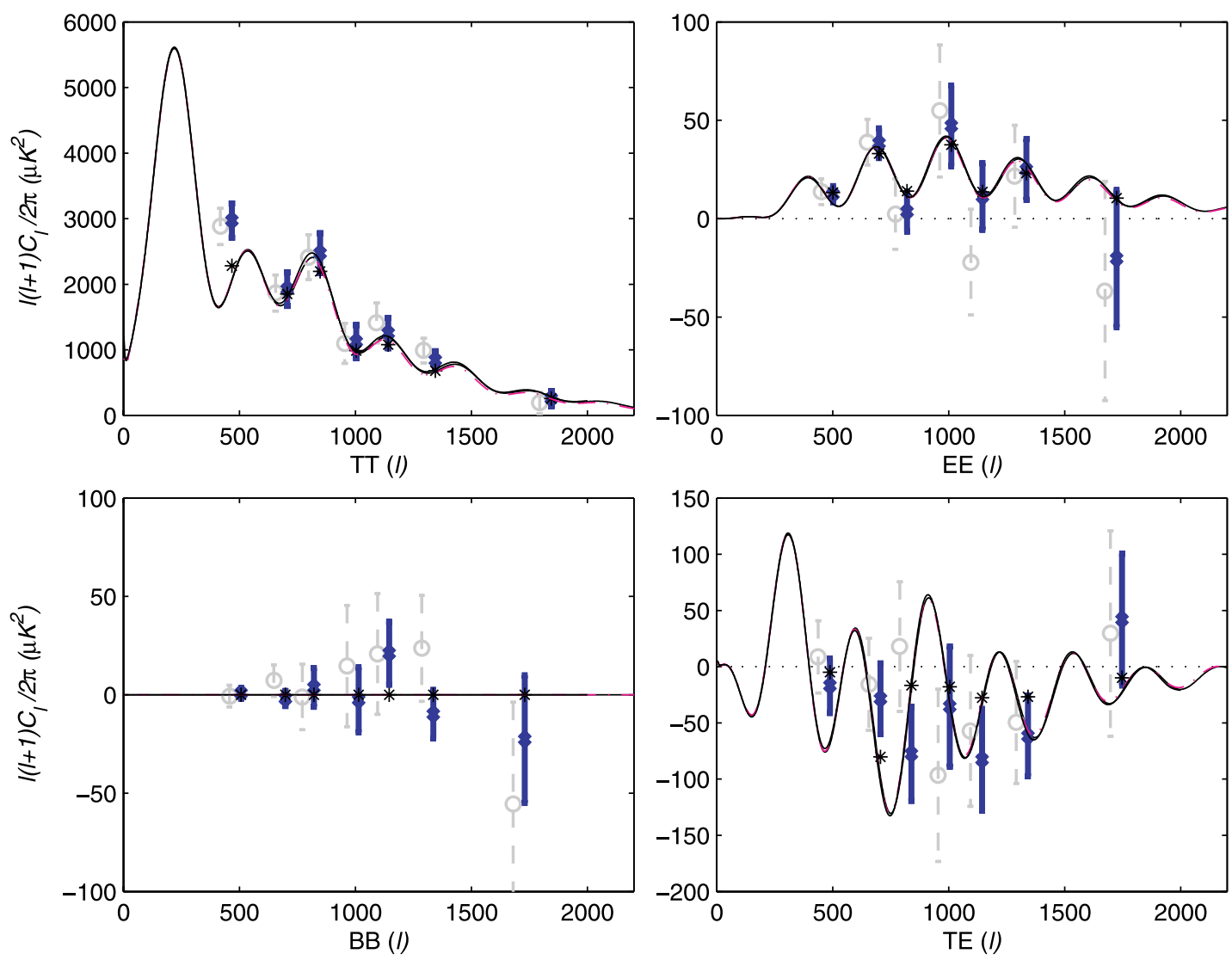

FIG. 1.-CBI maximum likelihood bandpowers and their inverse Fisher matrix errors. The blue crosses show power spectra $\mathcal{C}_{l}^{X}$ as a function of multipole $l$, derived from the CBI 2002 September-2005 April data, for the total intensity TT, the grad polarization EE, the curl polarization BB, and the cross-correlation TE. The magenta dashed curve represents a flat power-law $\Lambda \mathrm{CDM}$ model that best fits the $W M A P, \mathrm{CBI}$, and ACBAR CMB data (Spergel et al. 2003). It is very near the black fiducial $\mathcal{C}_{l}^{X(s)}$ that best fits the WMAP1+CBI+DASI+B03 TT+EE+TE data, whose parameters are given in Table 2. The black asterisks show the expected values of the bandpowers calculated from the fiducial model using the window functions. The $\chi^{2}$ values of the data relative to the fiducial model are 13.1, 2.25, 2.90, and 8.43 for TT, EE, BB, and TE, respectively, for 7 degrees of freedom. As expected, the BB spectrum is consistent with zero, with a single-band amplitude of $0.2 \pm 1.6 \mu \mathrm{K}^{2}$ and a $95 \%$ upper limit of $3.76 \mu \mathrm{K}^{2}$. (The gray circles show the results of Readhead et al. [2004b] for comparison. The shrinking in error bars is primarily due to the $54 \%$ increase in the data.) The CBI's low- $l(l \lesssim 360)$ response is set by the details of the sidelobes of the primary beam, which are difficult to measure; analysis with finer bins shows that the apparent discrepancy in the first TT bin plotted here is confined to $l<360$, where the wings of the primary beam are picking up the first Doppler peak. Consequently we neglect the $l<360$ TT from CBI in further analysis. The data have been offset in $l$ for clarity.

uncertainty of $1.3 \%$ in flux density (Readhead et al. 2004b). Six of the antennas were set to receive right-circular polarization $(R)$ and seven left $(L)$, so each visibility measurement represents one of the four polarization products RR, RL, LR, or LL. The copolar products RR and LL are sensitive to total intensity or brightness temperature $T$ (under the assumption that the circular polarization $V$ is zero, as expected), while RL and LR are sensitive to linear polarization, which can be divided into grad-mode $E$ and curlmode $B$ components. In the small-angle approximation, where the celestial sphere can be described by a tangent plane ("image" or "sky" plane), the angular spherical harmonic multipoles defining the CMB radiation field, labeled by $(l, m)$, become Cartesian components of $l,\left(l_{u}, l_{v}\right)$. In this same approximation, interferometer visibilities sample the Fourier transform of the sky brightness, with $(u, v)$ as the conjugate variables to angles on the celestial plane; this " $(u, v)$-space" is related to " $l$-space" by $\left(l_{u}, l_{v}\right)=2 \pi(u, v)$. Each interferometer baseline is therefore sensitive to emission on angular scales centered on the spherical harmonic multipole $l=2 \pi x$, where $x$ is the antenna separation in wavelengths.

The visibilities are processed by convolution with an $l$-space gathering kernel to produce gridded estimators $\Delta_{i Q}$ for polariza-

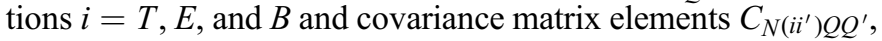
$\left.C_{T\left(i^{\prime}\right)}\right) Q Q^{\prime}$, and $C_{P\left(i i^{\prime}\right) Q Q^{\prime}}$ for (Gaussian) instrumental noise, (Gaussian) $\mathrm{CMB}$ signals, and projection templates associated with point sources and ground spillover (Myers et al. 2003; Readhead et al. 2004b). This gridding compresses the $\sim 10^{7}$ visibilities to $\sim 10^{4} l$-space "pixels" (labeled by $Q$ ) without loss of essential information.

For calculation of angular power spectra, the gridded estimators and covariance matrices are passed to a maximum likelihood procedure that estimates the CMB polarization bandpowers $q_{X b}$ in band $b$, where $X=\left(i i^{\prime}\right)=\mathrm{TT}$, EE, TE, and BB; associated noise bandpowers $q_{N X b}$; a Fisher (or likelihood curvature) matrix $F_{X b, X^{\prime} b^{\prime}}$, whose inverse encodes the variance around the maximum likelihood; and $l$-space window functions $W_{X b l}$. The $q_{X b}$ are defined by the expansion of the spectra $\mathcal{C}_{l}^{X}=\sum_{b} q_{X b} \mathcal{C}_{l b}^{X}$. [Here $\mathcal{C}_{l}^{X} \equiv$ $l(l+1)\left\langle a_{i l m} a_{i^{\prime} l m}^{*}\right\rangle / 2 \pi$, where $a_{i l m}$ denotes the multipole coefficients of the signal $s_{i}$, with $i=T, E$, and $B$.] To determine bandpowers, we use (theory-blind) flat $\mathcal{C}_{l b}^{X}$ shapes with top-hat binning (unity inside and zero outside of the band). The window functions from the pipeline convert the theoretical power spec$\operatorname{tra} \mathcal{C}_{l}^{X}$ into bandpowers $q_{X b}=\sum_{l} \mathcal{C}_{l}^{X} W_{X b l}(l+1 / 2) /[l(l+1)]$ to compare with the observed ones.

\subsection{The Power Spectra}

Figure 1 shows the CBI maximum likelihood bandpowers and their inverse Fisher matrix errors. The numerical values are given in Table 1. To minimize band-to-band correlations, only seven bands are shown for EE, but we use about twice as many bands 
TABLE 1

CBI Power Spectra in Seven Bands

\begin{tabular}{|c|c|c|c|c|}
\hline$l$-range & TT & $\mathrm{EE}$ & $\mathrm{TE}$ & BB \\
\hline$<600 \ldots \ldots$ & $2971 \pm 260$ & $12.5 \pm 3.9$ & $-16.9 \pm 24.4$ & $0.8 \pm 2.8$ \\
\hline $600-750 \ldots \ldots \ldots \ldots \ldots \ldots \ldots \ldots \ldots \ldots$ & $1925 \pm 252$ & $38.3 \pm 7.6$ & $-28.5 \pm 31.3$ & $-1.9 \pm 4.0$ \\
\hline 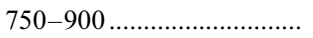 & $2475 \pm 304$ & $3.6 \pm 9.9$ & $-77.6 \pm 41.9$ & $3.8 \pm 11.0$ \\
\hline 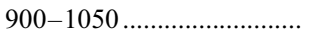 & $1126 \pm 248$ & $47.2 \pm 20.6$ & $-35.5 \pm 53.7$ & $-2.6 \pm 17.2$ \\
\hline 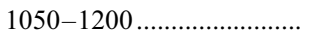 & $1256 \pm 239$ & $11.3 \pm 17.8$ & $-82.8 \pm 45.7$ & $21.1 \pm 16.1$ \\
\hline 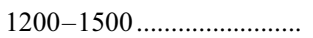 & $841 \pm 137$ & $25.0 \pm 15.2$ & $-61.7 \pm 35.7$ & $-9.9 \pm 12.0$ \\
\hline$>1500$ & $256 \pm 118$ & $-20.3 \pm 35.4$ & $42.0 \pm 59.6$ & $-22.6 \pm 31.6$ \\
\hline
\end{tabular}

NotE.-The quantity $\mathcal{C}_{l}$ is given in units of $\mu \mathrm{K}^{2}$.

for our cosmic parameter analyses. A nonlinear transformation to a Gaussian in the "offset lognormal" combination $\ln \left(q_{X b}+q_{N X b}\right)$ is used to give a more accurate representation of the bandpower likelihood surface (Bond et al. 2000; Sievers et al. 2003). Figure 2 shows the spectra of pairs of fields of the CBI data (which have better sensitivity than individual fields). These demonstrate that the remarkably good agreement between the CBI EE spectrum and the fiducial model for EE that is evident in Figure 1 is due to random chance.

If the overall amplitude of $C_{P}$ is set to a large value, then the "nuisance" modes represented in the construction of the matrix are projected out. This is essential for ground subtraction and point-source projections for $T$. As discussed in Readhead et al. (2004b), where we project out the brightest $\sim 20 \%$ of the NRAO VLA Sky Survey (NVSS) sources in $E$ and $B$, we see no evi- dence for any polarized point sources in the $\mathrm{CBI}$ data. In particular, for $\mathrm{CBI}$ the EE spectrum is strongly detected, while the BB spectrum is consistent with zero, whereas uncorrelated polarized point sources give rise to (roughly) equal amounts of power in $\mathrm{EE}$ and BB. Neither polarization power spectrum rises like $l^{2}$, as would be expected from an appreciable point-source signal. The spectra with and without the brightest $\sim 20 \%$ of NVSS sources projected out in polarization are very similar, with no systematic trend in the differences. Finally, there are no sources visible in the CBI polarization maps. Consequently, in this work, we do not project out any sources in polarization. The nature of the $30 \mathrm{GHz}$ polarized radio source population is currently poorly known, but some models have been devised on the basis of extrapolation from lower frequency surveys. These generally indicate that contamination of the EE and BB power spectra should be negligible
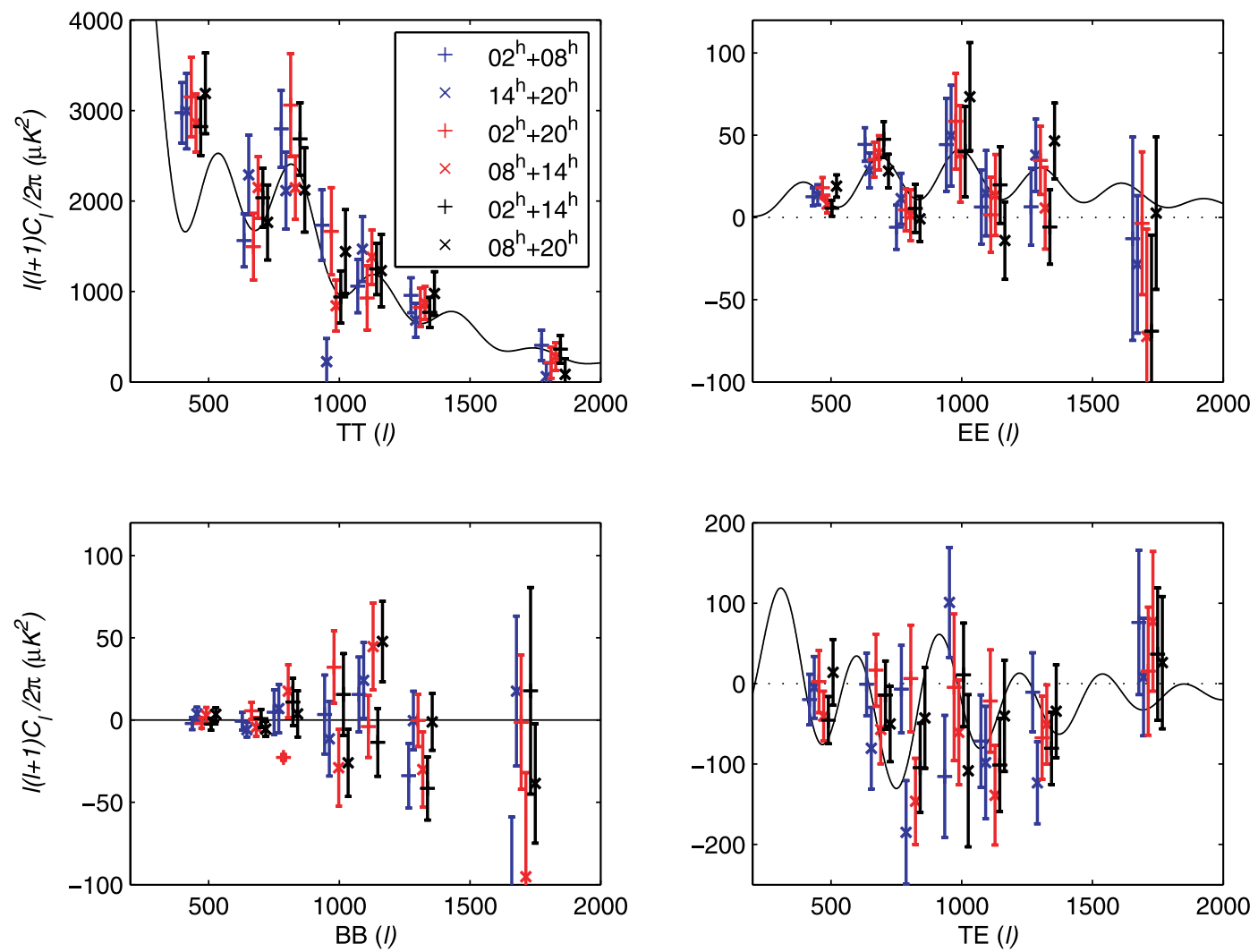

FIG. 2.-Power spectra for the six distinct pairs of CBI fields, each denoted by the colors and symbols indicated in the TT panel. For example, the blue plus signs

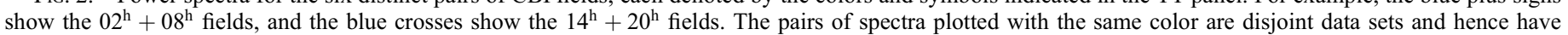

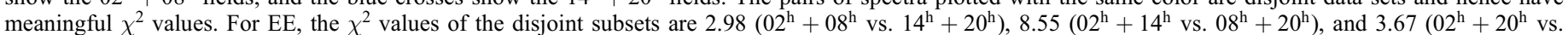

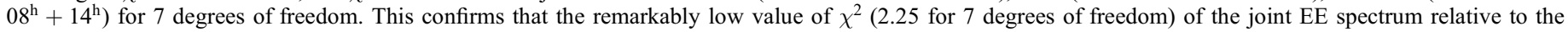
fiducial model is due to random chance. The points have been spread in $l$ about the bin center for clarity. 
E: $0<<1000$

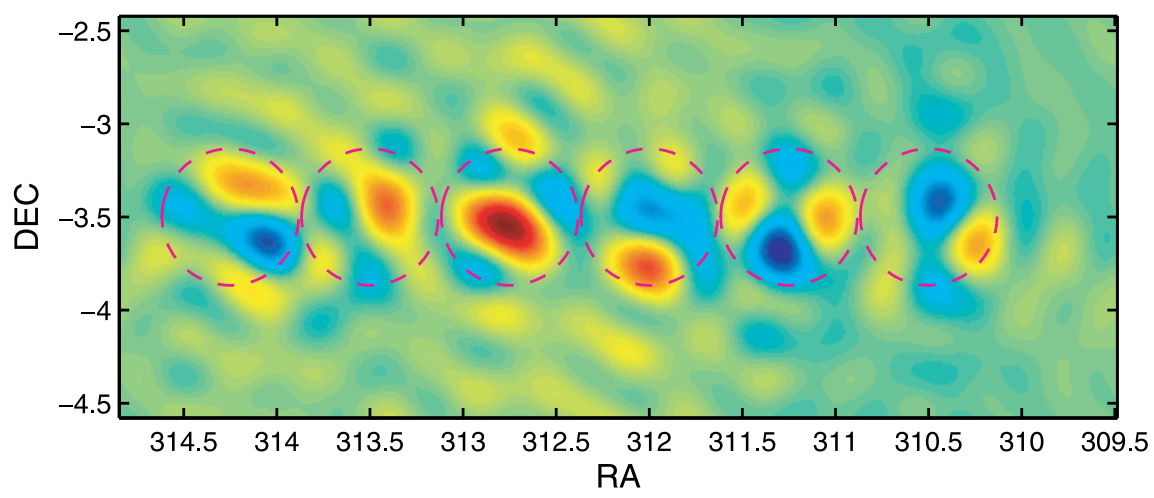

B: $0<<1000$

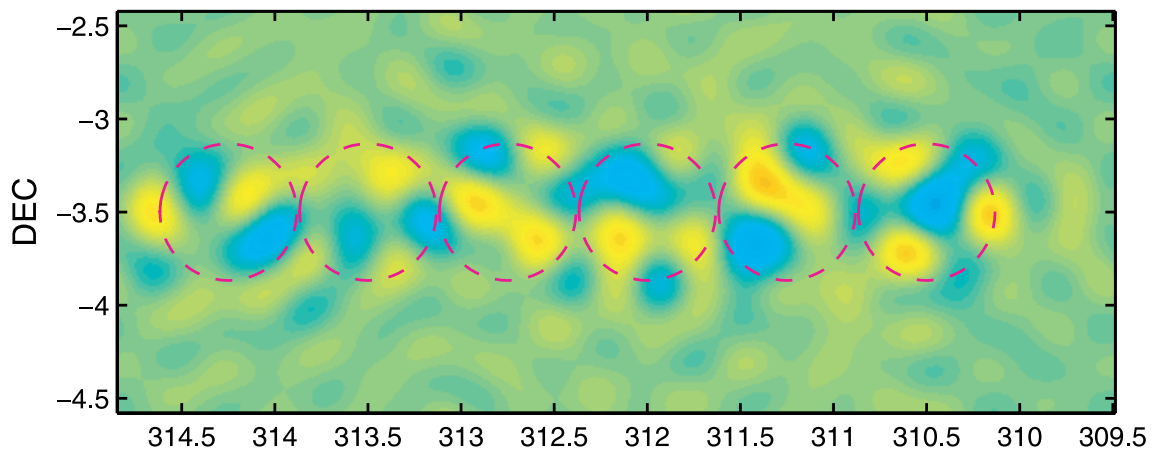

RA
mJy/beam

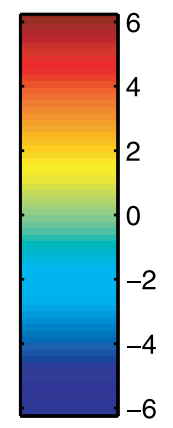

mJy/beam

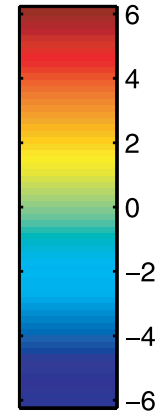

(a)

(b)

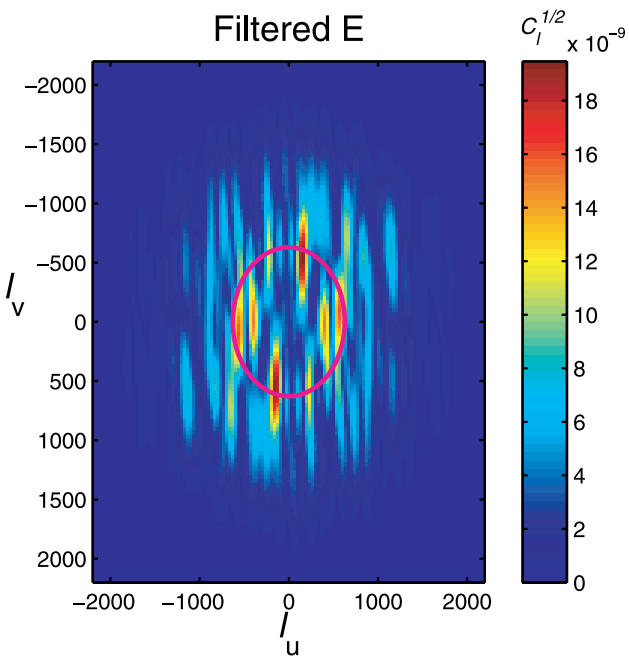

(c)
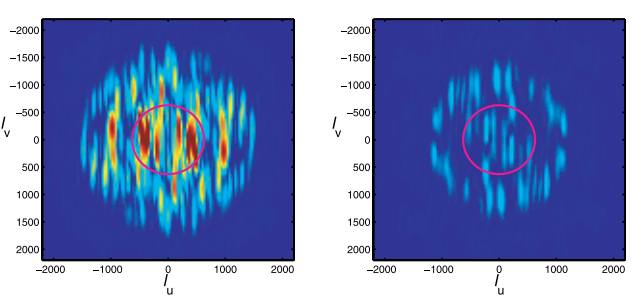

(d)
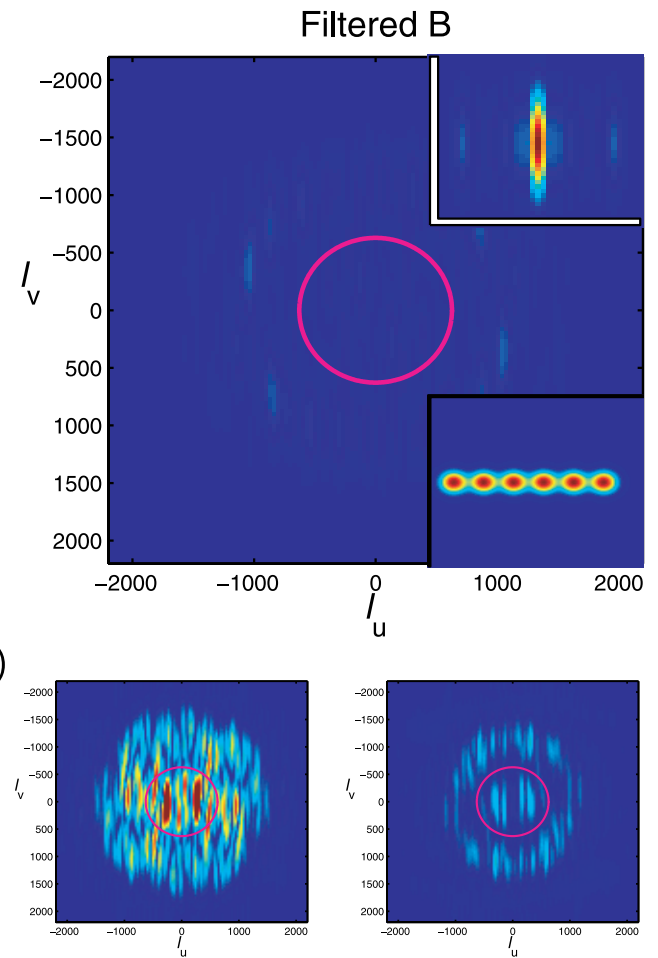

FIG. 3.- (a) Raw images of the (approximate) $E$ and $B$ signals from the $20^{\mathrm{h}}$ deep strip for $l<1000$. The circles show the FWHM of each of the six CBI pointings in the strip. A filter has been applied to remove the mean-mode (ground) signal, which would otherwise dominate the maps. No other filters are needed: the raw $20^{\mathrm{h}}$ data are dominated by the CMB signal. For the data plotted here, simulations of pure $E$ and pure $B$ signals show that the mixing between $E$ and $B$ from sky coverage is $\sim 5 \%$ in variance. The $E$ map, which is predominantly signal, has a variance 2.8 times larger than that in the $B$ map (which is consistent with noise). Because Fourier transforms preserve power, the $E / B$ variance ratio is the same in $l$-space. (b) Modulus of the optimal (Wiener-filtered) signal maps seen in the gridded $\left(l_{u}, l_{v}\right)$-plane using our signal reconstruction process, where $\left(l_{u}, l_{v}\right)=2 \pi(u, v)$. The maps have been normalized to $C_{l}^{1 / 2}$, equivalent to plotting $a_{l, m}$ for a full-sky map. The radius of the magenta circles is $l=630$, the peak of the CBI's sensitivity. The EE/BB power ratio in the Wiener-filtered, reconstructed maps is 14.8 . The upper inset in the $B$ map is the average mosaic smoothing kernel $\mathcal{B}$ that we chose to smooth the reconstructed maps. The strong anisotropy in $l_{u}, l_{v}$ reflects the geometry of the $20^{\mathrm{h}}$ strip. The lower inset is the sky-plane representation of $\mathcal{B}$. $(c, d)$ Two sample maps of the fluctuations $\delta s=s-\langle s \mid \Delta\rangle$, given on the same scale as $(b)$. 
(a)
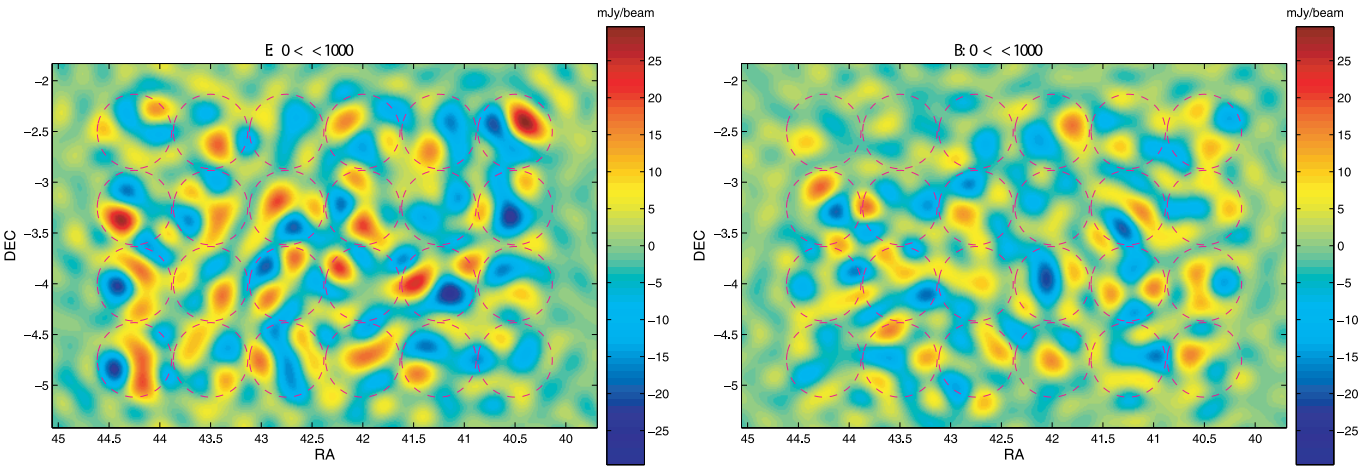

(b)
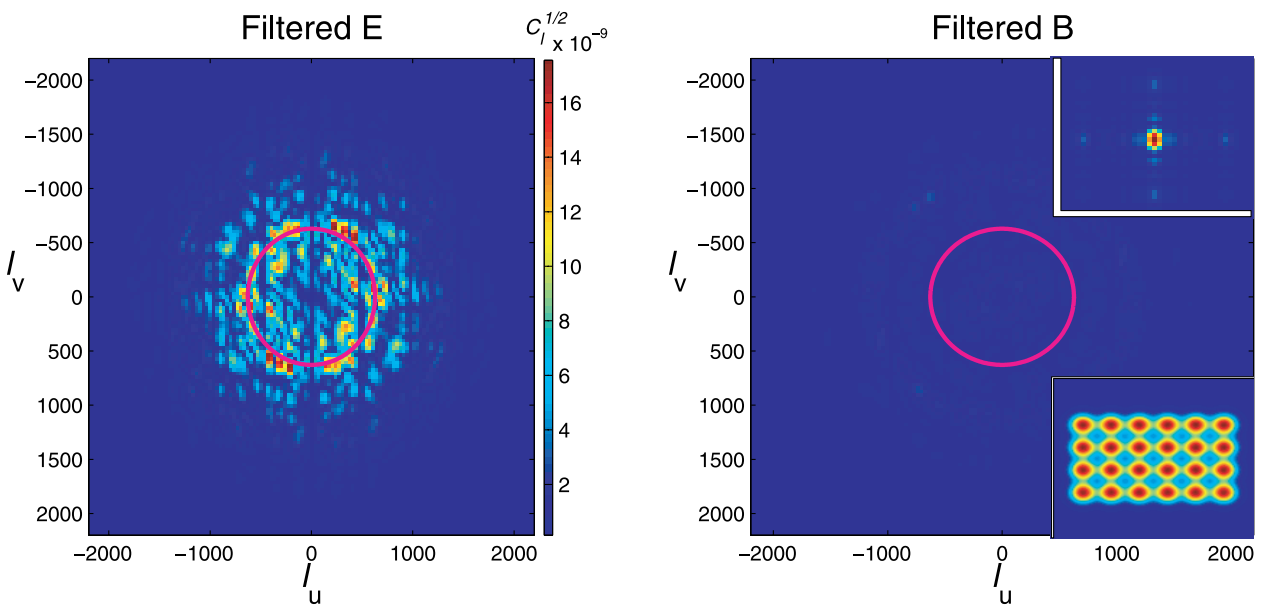

(c)
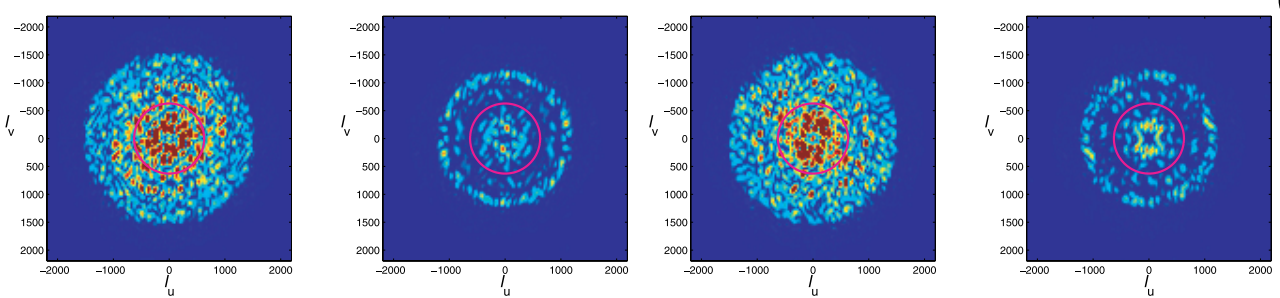

(d)

Fig. 4.-(a) Raw maps of the (approximate) $E$ and $B$ signals from the $02^{\mathrm{h}}$ mosaic field for $l<1000$. The two northern strips in this field have substantially less data than the four southernmost strips; to keep the noisy northern strips from visually dominating the map, they have not been included in the maps. A filter has been applied to remove the mean-mode (ground) signal, which would otherwise dominate the maps. The variance in the $E$ map is 1.71 times larger than the variance in the $B$ map (which is consistent with the noise). The circles show the FWHM of each of the 24 CBI pointings used in the maps (all 36 mosaic pointings are used in producing the spectrum). (b) Modulus of the optimal (Wiener-filtered) signal maps seen in the gridded $\left(l_{u}, l_{v}\right)$ plane using our signal reconstruction process, where $\left(l_{u}, l_{v}\right)=2 \pi(u, v)$. The maps have been normalized to $C_{l}^{1 / 2}$, equivalent to plotting $a_{l, m}$ for a full-sky map. The radius of the magenta circles is $l=630$, the peak of the CBI's sensitivity. The striping apparent in the $E$ image is due to the ground filter removing Fourier modes equal to the separation of the mosaic fields in right ascension. The EE/BB power ratio is 24. The ring at $l \sim 1000$ in the $B$ image is due to the $\sim 1.3 \sigma$ bandpower value in the fifth BB bin that enters into the filter. The upper inset in the $B$ map is the average mosaic smoothing kernel $\mathcal{B}$ that we chose to smooth the reconstructed maps. The strong anisotropy in $l_{u}, l_{v}$ in Fig. 3 is not there in this square map. The compactness of $\mathcal{B}$ relative to that of Fig. 3 demonstrates the improved $l$-space resolution of a mosaic, at a cost of the signal-to-noise ratio. The cross pattern extending from the central peak of $\mathcal{B}$ arises from the Fourier transform of the mosaic pattern on the sky. The lower inset shows the sky-plane representation of $\mathcal{B}$. $(c, d)$ Two sample realizations of the fluctuations $\delta s=s-\langle s \mid \Delta\rangle$, given on the same scale as $(b)$.

for the CBI $l$-range, observing frequency, and sensitivity levels (e.g., Tucci et al. 2004). Further discussion of this issue will be presented in S. T. Myers et al. (2007, in preparation).

\subsection{Raw Maps and Signal-reconstructed Maps}

The gridded estimators allow us to efficiently reconstruct the polarization of the CMB. We use the fact that $E$ and $B$ are real field components that completely describe the polarization field to present $E$ and $B$ sky images in Figures $3 a$ and $4 a$. These images are created by Fourier-transforming the ground-filtered $l$-space estimators $\Delta_{E Q}$ and $\Delta_{B Q}$. These $\Delta_{E Q}$ and $\Delta_{B Q}$ in turn are convolved representations of the true $l$-space $\tilde{E}$ and $\tilde{B}$, which are re- lated to the linear polarization Stokes parameters $Q$ and $U$ (Kovac et al. 2002). These novel $E$ and $B$ images and $l$-space maps differ from the traditional polarization "headless" vector plots based on decomposing the images in Stokes $Q$ and $U$ into $E$-like and $B$-like components, as they are direct representations of the $E$ and $B$ fields and their transforms. Note that due to the nonlocal transformation relating $E$ and $B$ to the $Q$ and $U$ fields on the sky (e.g., Lewis et al. 2002; Bunn et al. 2003), the $E$ and $B$ images do not show polarized "objects" but rather coherences in the polarization field.

The dominant ground contamination has been removed from the estimators by forming $C_{N}\left(C_{N}+C_{P}\right)^{-1} \Delta$ in the large- $C_{P}$ 

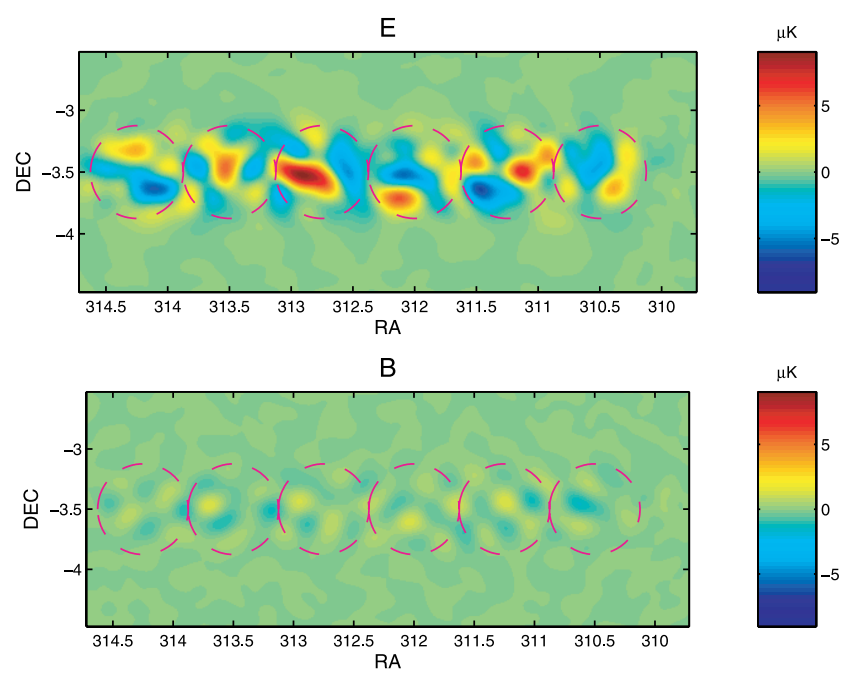

FIG. 5.- Reconstructed $E$ and $B$ signal sky-plane images of the $20^{\mathrm{h}}$ deep strip, the transform of the $l$-space images in Fig. $3 b$. These should be compared with the raw images in Fig. $3 a$.

limit. Here $C_{N}$ serves to regularize $C_{P}$. Other regularizers, such as $C_{N}+C_{T}$, give virtually identical results. The images generated from the estimators include the effects of the observing strategy and of the gridding process, which include the mosaic pattern, $(u, v)$-plane coverage, the primary beam, and noise weighting, similar to a standard interferometric "dirty map." Hence, the raw images are not faithful reconstructions of the true intensity and polarization. Furthermore, the Fourier transform of the regular mosaic pattern introduces "sidelobes" in the $l$-space map made from the raw estimators. Therefore, filtering and deconvolution can be beneficial to our maps.

Figures $3 b$ and $4 b$ show optimal Wiener filter $l$-space maps. These are smoothed mean signals given the observations,

$$
\langle s \mid \Delta\rangle=\mathcal{B} \mathbf{R}^{\dagger}-1 C_{T} C_{\text {tot }}^{-1} \Delta, \quad C_{\text {tot }} \equiv C_{N}+C_{P}+C_{T} .
$$

Here $\mathcal{B}$ is a smoothing kernel, and the matrix $\mathbf{R}$ takes signal space into data space, with $\Delta=\mathbf{R} s+n$, where $s$ is the signal and $n$ is the map noise, including ground and source projection terms. One is free to choose the basis in which to describe the true sky signals $s_{i}$; for example, as Stokes parameters on the sky relative to a fixed sky basis or as a set of $a_{i l m}$ coefficients or (in the smallangle limit) as the set of related Fourier transform coefficients. For the $\mathrm{CBI}$, and for interferometers in general, it is natural to express the $s_{i}$ in the $l$-basis, as in Figures $3 b$ and $4 b$, but we also show the sky-plane version in Figure 5. The $C_{T}^{X}=\sum q_{X b} C_{T b}^{X}$ used in the signal reconstructions are derived from the data using the measured bandpowers $q_{X b}$ (set to zero if negative) and the top-hat band matrices $C_{T b}^{X}$ and hence are "theory-blind."

Typically the signal-space dimension would be larger than the data-space dimension, so $\mathbf{R}$ would not be invertible. By modeling the $\left(l_{u}, l_{v}\right)$-plane only at points where we have estimators, the two dimensions are equal, and $\mathbf{R}$ becomes square and is, in principle, invertible. In practice, $\mathbf{R}$ has an enormous condition number, so we remove poorly measured modes with eigenvalues of less than $10^{-4}$ times the largest eigenvalue of $\mathbf{R}$ when we construct the $\mathbf{R}^{-1}$ operator. This makes the reconstruction better conditioned, and the results are insensitive to variations in the eigenvalue cut. The remaining "noise" in $\mathbf{R}^{-1}$ is controlled by reconvolution with a smooth regularizer $\mathcal{B}$.
We have freedom in the choice of the smoothing kernel $\mathcal{B}$. The one we choose for the images is a natural one associated with the map. If we let a pixel be denoted by the vector $\boldsymbol{Q}=$ $\left(l_{u}, l_{v}\right)$, the matrix $\mathbf{R}$ has components $\mathbf{R}_{\boldsymbol{Q}, \boldsymbol{Q}+\boldsymbol{\delta} \boldsymbol{l}}$, where the vector $\boldsymbol{\delta} \boldsymbol{l}$ goes over the region of $l$-space that contributes to the pixel in question. The matrix $\mathbf{R}$ tends to be only roughly independent of $\boldsymbol{Q}$. We take our smoothing kernel $\mathcal{B}_{\delta l}$ to be the average of $\mathbf{R}_{\boldsymbol{Q}, \boldsymbol{Q}+\boldsymbol{\delta} \boldsymbol{l}}$ over all pixels $\boldsymbol{Q}$. As can be seen in the insets in Figures $3 b$ and $4 b$, this is very nearly the Fourier transform of the mosaic pattern on the sky, as is required to make the sky-plane images reflect the area actually observed. For the strip, $\mathcal{B}_{\delta l}$ is quite asymmetric, as shown in Figure 3. For the other three square CBI polarization fields, the $\mathcal{B}_{\delta l}$ is nearly symmetric, as shown in Figure 4 . There are low-level "sidelobes" in the $l_{u}$ - and $l_{v}$-directions due to the mosaic spacing of $45^{\prime}$. Figure 5 shows the sky-plane (as opposed to $l$-space) representation of the reconstructed signal maps for the deep strip. The agreement between the raw $E$ image in Figure $3 a$ and the reconstructed $E$ image in Figure 5 is quite good.

To assess how well the mean field $\langle s \mid \Delta\rangle$ describes the actual distribution of signal on the sky, it is important to see how large the fluctuations $\delta s \equiv s-\langle s \mid \Delta\rangle$ are about it. For Gaussian signals, the statistics of the (smoothed) $\delta s$ are fully described by the constrained correlation matrix

$$
\begin{aligned}
\left\langle\delta s \delta s^{\dagger} \mid \Delta\right\rangle & =\mathcal{B}\left(w_{S}^{-1}-w_{S}^{-1} \mathbf{R}^{\dagger} C_{\text {tot }}^{-1} \mathbf{R} w_{S}^{-1}\right) \mathcal{B}^{\dagger} \\
& =\mathcal{B}\left[w_{S}+\mathbf{R}^{\dagger}\left(C_{N}+C_{P}\right)^{-1} \mathbf{R}\right]^{-1} \mathcal{B}^{\dagger}
\end{aligned}
$$

Here $w_{S}^{-1}$ is the correlation matrix of $s$, the unconstrained signal in signal space. The signal variance in data space is therefore $C_{T}=\mathbf{R} w_{S}^{-1} \mathbf{R}^{\dagger}$. In terms of $w_{S}$, the unsmoothed mean field is $\langle s \mid \Delta\rangle=w_{S}^{-1} \mathbf{R}^{\dagger} C_{\text {tot }}^{-1} \Delta$. These equations apply to a general $\mathbf{R}$; for example, when the signal-space and data-space dimensions are unequal. If $\mathbf{R}$ is an invertible square matrix, then the smoothed mean field is given by equation (1) and the smoothed realizations of the fluctuations are of the form

$$
\begin{aligned}
\delta s & =\left\langle\delta s \delta s^{\dagger} \mid \Delta\right\rangle^{1 / 2} g \\
\left\langle\delta s \delta s^{\dagger} \mid \Delta\right\rangle & =\mathcal{B} \mathbf{R}^{-1}\left(C_{T}-C_{T} C_{\text {tot }}^{-1} C_{T}\right) \mathbf{R}^{\dagger-1} \mathcal{B}^{\dagger}
\end{aligned}
$$

Here $g_{i Q}$ are independent Gaussian random variables with unit variance and $\left\langle\delta s \delta s^{\dagger} \mid \Delta\right\rangle^{1 / 2}$ is the matrix square root. Equation (4) shows that the fluctuations go to zero in modes in which the generalized noise is small, but approach pure signal realizations in modes in which it is high. Figures $3 c, 3 d, 4 c$, and $4 d$ show a few examples using the $C_{T}$ derived for the four CBI fields. These illustrate that the reconstructed signal for the deep strip is better determined than that for the mosaic fields.

We have also used a modified version of the CLEAN deconvolution algorithm (Högbom 1974) to do the effective inversion of $\mathbf{R}$ : we find the largest signal among the $(u, v)$-plane estimators $\Delta_{i Q}$; we place a $\delta$-function in $l$-space there that zeroes out the $\Delta_{i Q}$; we subtract from each estimator its response to that signal; we then repeat, ending only when $\sim 10^{4}$ components have been found. This leads to an error in the residual of less than $10^{-5}$ times the power in the original. This method has several nice features: since each estimator has compact support in the $(u, v)$ plane, the process is quite stable because the addition of a model component only affects a few estimators; since the model is in the same space as the data, no (time-consuming) Fourier transforms or (expensive) decompositions of $\mathbf{R}$ are needed. As in the standard interferometer imaging application of the CLEAN algorithm, a smooth restoring convolution kernel $\mathcal{B}$ is required to turn the set 


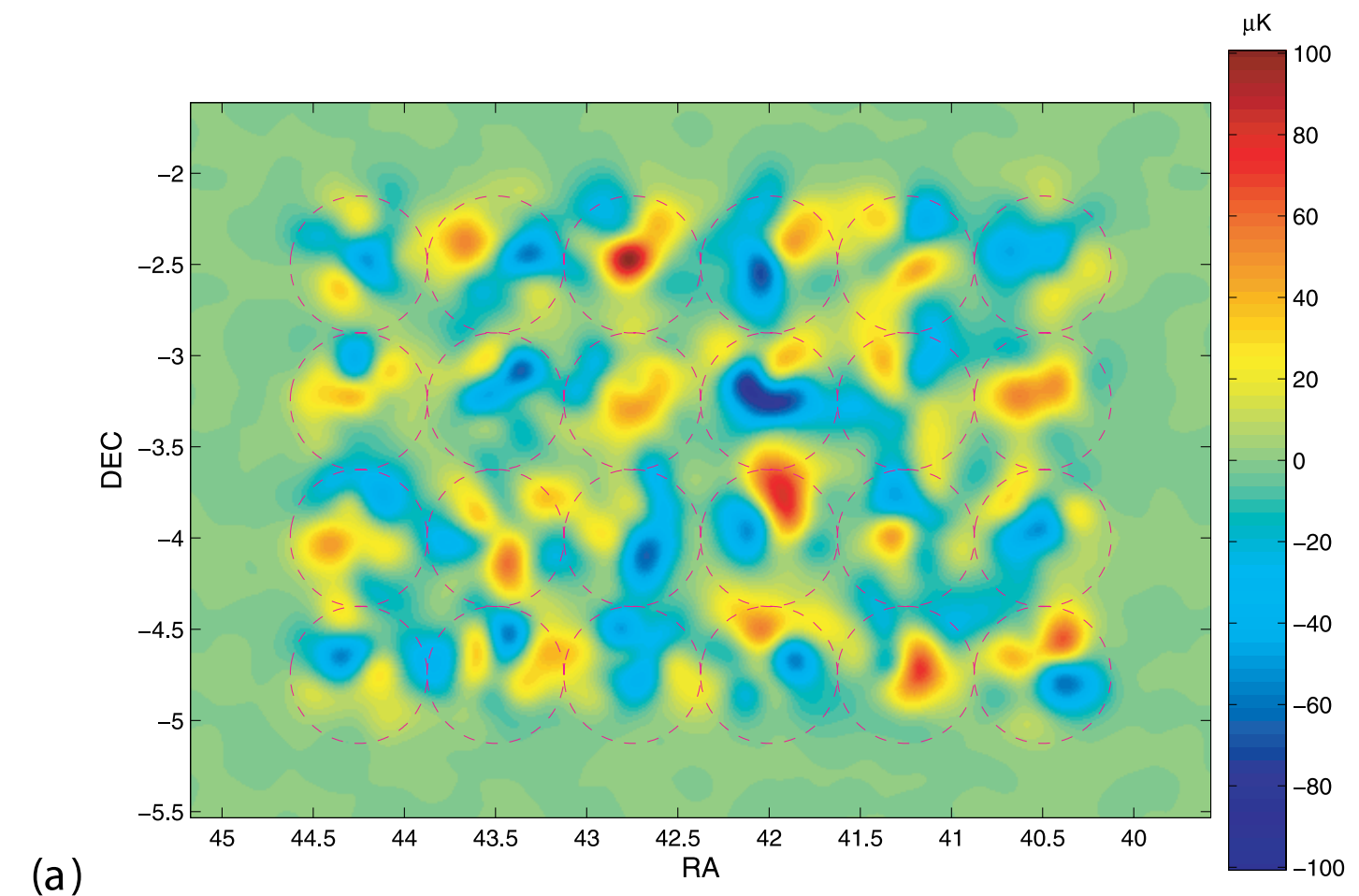

(b)
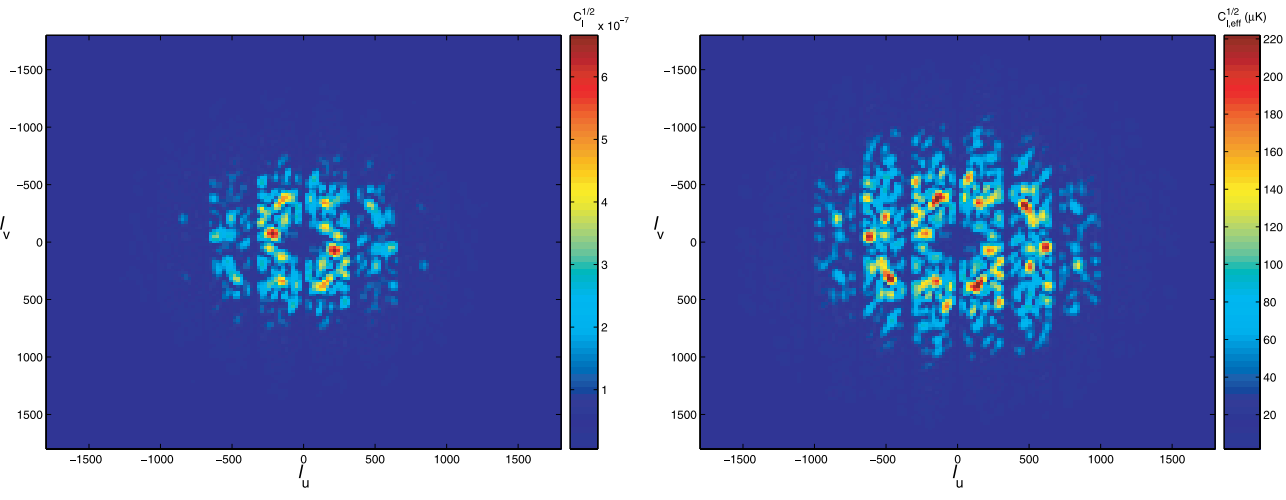

(c)

FIG. 6. - (a) Reconstructed sky image for the Wiener-filtered $02^{\mathrm{h}}$ mosaic in total intensity. Both ground and sources (which would otherwise dominate the map) have been removed as part of the filter. This image has been produced using the $(u, v)$-plane CLEAN procedure described in $\S 2.3$. The circles show the FWHM of each of the 24 CBI pointings used in Fig. 4. (b) Modulus of the optimal (Wiener-filtered) map of $(a)$ in the gridded $\left(l_{u}, l_{v}\right)$ plane. As in Fig. $4 b$, the vertical striping is due to the ground filter. (c) Rescaling of $(b)$ that brings out the structure in the $\left(l_{u}, l_{v}\right)$-plane by multiplying each pixel by $l$. This is equivalent to using $l(l+1) C_{l} / 2 \pi$ instead of $C_{l}$ to plot the angular power spectrum. In this representation, one can see hints of multiple rings of higher signal that correspond to the Doppler peaks in the power spectrum.

of $\delta$-functions in $l$-space resulting from the CLEANing into a smooth transformable map; otherwise, the image would have artifacts.

We find that the results using eigenvalue cuts or this CLEAN method give very similar maps. The sky-plane and $l$-space total intensity images of the $02^{\mathrm{h}} \mathrm{CBI}$ mosaic that we display in Figure 6 were constructed using this CLEAN algorithm. As expected, given the relatively small errors on the $T$ bandpowers, all four fields show strong $T$ detections.

\section{PARAMETERIZED POLARIZATION PHENOMENOLOGY}

\subsection{TT, EE, TE, and BB Bandpower Data}

In our parameter determinations we consider five combinations of bandpower data: (1) the CBI TT+EE+TE bandpowers obtained from the analysis described here of the 2002-2005 data with a bin width of $\Delta l \approx 75$, more fine-grained than those of Table 1 (available as an online supplement); (2) CBI $l=600$ 1960 TT bands from the combined mosaic and deep field analysis of Readhead et al. (2004a); (3) TT and TE WMAP1 bandpowers from the first year of WMAP data ${ }^{12}$ (Bennett et al. 2003), adopting the likelihood mapping procedure described in Verde et al. (2003); (4) DASI TT (Kovac et al. 2002) and 3 yr EE+TE (Leitch et al. 2005) results; and (5) the recent BOOMERANG (hereafter B03) TT+EE+TE results and (Masi et al. 2005; Jones et al. 2006; Montroy et al. 2006; Piacentini et al. 2006), with $l<300$ TT bandpowers excluded because of overlap with WMAP (although this has no quantitative impact). We also omit CBI TT results for $l<360$, both because of the overlap with WMAP and because the (very limited) sensitivity there comes from the sidelobes of the primary beam, which are extremely difficult to measure accurately. The

\footnotetext{
12 None of the conclusions drawn here are affected by using the WMAP $3 \mathrm{yr}$ power spectrum (which was released after the submission of these results).
} 
TABLE 2

Cosmic Parameter Values for the Flat Tilted Adiabatic $\Lambda$ CDM Model

\begin{tabular}{|c|c|c|c|c|}
\hline $\begin{array}{l}\text { Parameter } \\
\text { (1) }\end{array}$ & $\begin{array}{l}\text { Prior Range } \\
\text { (2) }\end{array}$ & $\begin{array}{c}\text { WMAP } 1+\mathrm{CBI}+\mathrm{DASI}+\mathrm{B} 03 \\
\mathrm{TT}+\mathrm{EE}+\mathrm{TE}(\text { Best Fit) } \\
\text { (3) }\end{array}$ & $\begin{array}{c}\text { WMAP } 1+\mathrm{CBI}+\mathrm{DASI}+\mathrm{B} 03 \\
\mathrm{TT}+\mathrm{EE}+\mathrm{TE} \\
\text { (4) }\end{array}$ & $\begin{array}{c}\mathrm{CBI}+\mathrm{DASI}+\mathrm{B} 03 \\
\mathrm{EE}+\mathrm{TE} \\
(5)\end{array}$ \\
\hline$\theta / \theta_{0}$ & $0.5-10$ & 1 & $1.001 \pm 0.0042$ & $0.986 \pm 0.017$ \\
\hline$\Omega_{b} h^{2} \ldots \ldots \ldots \ldots \ldots \ldots \ldots \ldots \ldots \ldots$ & $0.005-0.1$ & 0.0226 & $0.0232 \pm 0.0013$ & $0.018 \pm 0.005$ \\
\hline$\Omega_{c} h^{2} \ldots \ldots \ldots \ldots \ldots \ldots \ldots \ldots \ldots \ldots$ & $0.01-0.99$ & 0.117 & $0.114 \pm 0.011$ & $0.119 \pm 0.034$ \\
\hline$\tau$ & $0.01-0.8$ & 0.105 & $0.149 \pm 0.086$ & $0.33 \pm 0.18$ \\
\hline$n_{s}$ & $0.5-1.5$ & 0.960 & $0.978 \pm 0.039$ & $0.92 \pm 0.23$ \\
\hline $\ln \left(10^{10} A_{s}\right) \ldots \ldots \ldots$ & $2.7-4.0$ & 3.09 & $3.18 \pm 0.16$ & $3.37 \pm 0.35$ \\
\hline$q_{s}=A_{s} e^{-2 \tau} / A_{s 0} e^{-2 \tau_{0}} \ldots \ldots \ldots \ldots \ldots$ & $\ldots$ & 1 & $0.992 \pm 0.037$ & $0.86 \pm 0.14$ \\
\hline$\Omega_{\Lambda}$ & $\ldots$ & 0.714 & $0.733 \pm 0.054$ & $0.58 \pm 0.25$ \\
\hline 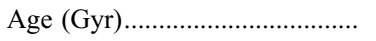 & $\ldots$ & 13.6 & $13.5 \pm 0.26$ & $14.4 \pm 0.80$ \\
\hline$\Omega_{m}$ & $\ldots$ & 0.286 & $0.267 \pm 0.054$ & $0.42 \pm 0.25$ \\
\hline$\sigma_{8}$ & $\cdots$ & 0.83 & $0.848 \pm 0.063$ & $0.94 \pm 0.21$ \\
\hline$z_{\mathrm{re}}$ & $\ldots$ & 12.5 & $15.1 \pm 5.3$ & $32 \pm 15$ \\
\hline$H_{0}$ & $40-100$ & 70.0 & $72.6 \pm 5.6$ & $64 \pm 15$ \\
\hline
\end{tabular}

NoTES.-The first six parameters are independent (fitted), and the last seven parameters are derived from them. Mean values and standard deviations are given for the TT+EE+TE data in col. (4) and for the EE+TE data in col. (5). The ranges for the uniform weak priors we imposed for the MCMC runs are given in col. (2). The best-fit model parameters defining our "fiducial model" are shown in col. (3). For this model, $\theta_{0}=1.0442$ and $A_{s 0} e^{-2 \tau_{0}}=18.0 \times$ $10^{-10}$. These are slightly different than the parameters defining the WMAP team's best fit (Spergel et al. 2003) using WMAP1 TT+TE data, plus ACBAR TT data, plus an earlier version of the CBI TT data (Pearson et al. 2003), and different priors: $\Omega_{b} h^{2}=0.0224, \Omega_{c} h^{2}=0.111, n_{s}(k=0.05)=0.958$, $\tau=0.11$, and $H_{0}=72$. This was the fiducial model used in Readhead et al. (2004b). Fig. 1 shows that the two are very similar visually.

Markov chain Monte Carlo (MCMC) package CosmoMC ${ }^{13}$ (Lewis \& Bridle 2002), modified to include polarization spectra, the cross-correlation between TT and EE spectra, and isocurvature modes, is used to calculate posterior probability distributions (including priors) for cosmic parameters.

\subsection{The Basic Flat Tilted Adiabatic $\Lambda$ CDM Model}

The simplest inflationary paradigm is characterized by six basic parameters: $\omega_{b} \equiv \Omega_{b} h^{2}$, the physical density of baryons; $\omega_{c} \equiv$ $\Omega_{c} h^{2}$, the physical density of cold dark matter; $\theta \equiv 100 l_{s}^{-1}$, parameterizing the angular scale $l_{s}^{-1}$ associated with sound crossing at decoupling, which defines the overall position of the peak-dip pattern; $\ln \left(10^{10} A_{s}\right)$, the logarithm of the overall scalar curvature perturbation amplitude $A_{s}$, which is the scalar curvature power spectrum $\mathcal{P}_{s}(k)$ evaluated at the pivot point $k_{n}=0.05 \mathrm{Mpc}^{-1} ; n_{s}$, the spectral index of the scalar perturbations, which is defined by $\mathcal{P}_{s}(k) \propto k^{n_{s}-1}$; and $\tau$, the Thomson scattering depth to decoupling. We do not consider gravitational wave-induced components.

Table 2 shows the broad priors that we have chosen for the basic parameter ranges so as to have little influence on our results. We also impose a weak- $h$ prior on the Hubble constant $H_{0}=100 h \mathrm{~km} \mathrm{~s}^{-1} \mathrm{Mpc}^{-1}: 0.4<h<1$. For the flat $\Omega_{\mathrm{tot}}=$ 1 models considered here, this weak- $h$ prior has little influence on the results, although some extreme models with high Thomson depth are excluded. The strongest prior is the flat restriction, which is expected in most inflation models. Some parameters change significantly when the flat prior is relaxed (Bond et al. 2003; Readhead et al. 2004a).

We highlight distributions for two parameters, the patternshifting $\theta / \theta_{0}$ and $q_{s}=A_{s} e^{-2 \tau} / A_{s 0} e^{-2 \tau_{0}}$, which determines the overall $\mathcal{C}_{l}^{X}$ amplitude. We normalize relative to $\theta_{0}=1.0442$ and $A_{s 0} e^{-2 \tau_{0}}=18.0 \times 10^{-10}$, the best-fit values for the WMAP1+ $\mathrm{CBI}+\mathrm{DASI}+\mathrm{B} 03 \mathrm{TT}+\mathrm{EE}+\mathrm{TE}$ data (Table 2). The near-degeneracy between $A_{s}$ and $\tau$ is only weakly broken at very low $l$, where reionization has some influence, and at higher $l$ through (nonlinear)

13 Available at http://cosmologist.info/cosmomc. secondary phenomena such as weak lensing or the SunyaevZel'dovich effect. However, the $q_{s}$ combination is reasonably well determined, although there are correlations with other parameters, such as $\omega_{b}, \omega_{c}$, and $n_{s}$, especially with polarization data only.

The relative positions of the peaks in the TT and EE spectra are "phase-locked" by the physics of the acoustic oscillations at photon decoupling, with the multipole of the $j$ th TT peak $\propto j \theta^{-1}$ and the multipole of the $j$ th EE peak $\propto(j+1 / 2) \theta^{-1}$. The TE cross-spectrum has double the number of peaks (Fig. 1). For the WMAP1+CBI+DASI+B03 TT+EE+TE data, we find $\theta / \theta_{0}=$ $1.001 \pm 0.0041$ and $q_{s}=0.996 \pm 0.037$. For the WMAP1+CBI data, $\theta / \theta_{0}=0.999 \pm 0.005$, to be contrasted with the $1.000 \pm$ 0.005 obtained in Readhead et al. (2004b) for this $\theta_{0}$. These results for the mean values and standard deviations are also very similar to those obtained with other CMB data combinations (e.g., Bond et al. 2003; MacTavish et al. 2006).

For the WMAP1+CBI+DASI+B03 TT+EE+TE data, the other basic cosmic parameters, after marginalization, have distributions as shown in Figure 7, with median values and $1 \sigma$ errors as given in Table 2. The best-fit parameters for the fiducial model are also given there. Using the WMAP1 TT+TE and CBI+DASI+B03 TT data gives very similar results: the inclusion of the current high$l$ polarization data has little impact on parameter values for this limited set.

For the CBI+DASI+B03 EE+TE data, we get $\theta / \theta_{0}=0.986 \pm$ 0.017 and $q_{s}=0.86 \pm 0.14$, in good agreement with the TT result. (A few other parameters are also moderately well constrained, but most are not, as shown in Table 2.) Our $\theta / \theta_{0}$ result is not affected if we relax the flat prior $(0.988 \pm 0.018)$. For the CBI EE, $\theta / \theta_{0}$ is $0.986 \pm 0.031$ and $q_{s}$ is $1.27 \pm 0.48$. For the CBI EE+ TE data, $\theta / \theta_{0}$ is $0.95 \pm 0.027$, and for the DASI+B03 EE+TE data, it is $1.03 \pm 0.030$. For the CBI BB data we obtain $\theta / \theta_{0}=$ $0.95 \pm 0.10$. This should be interpreted as essentially the limit that we would get from the prior probabilities alone. This shows that our $\theta / \theta_{0}$ results are data-driven rather than prior-driven.

\subsection{A Peak/Dip Pattern Test}

Single-band or broadband results using theoretically motivated $\mathcal{C}_{l b}^{X}$ shapes can also be produced by our pipeline. These allow 

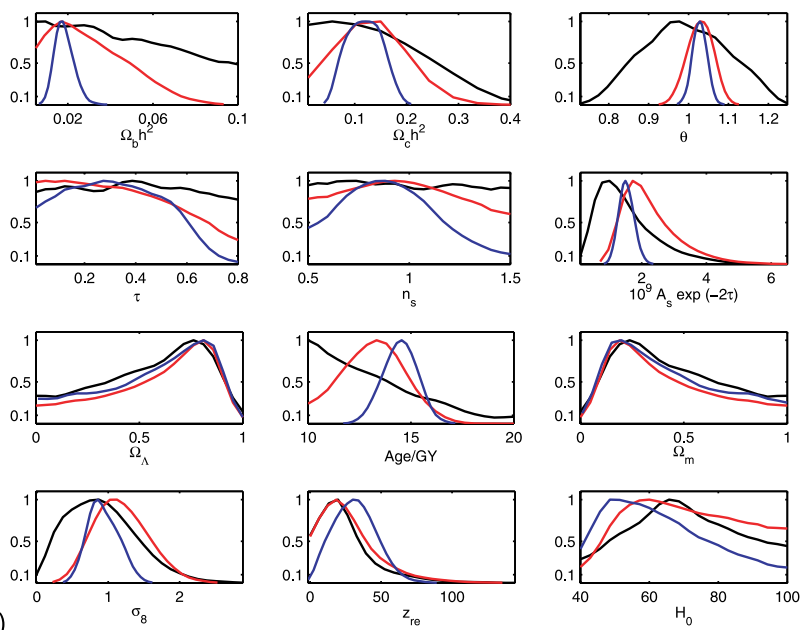

(a)
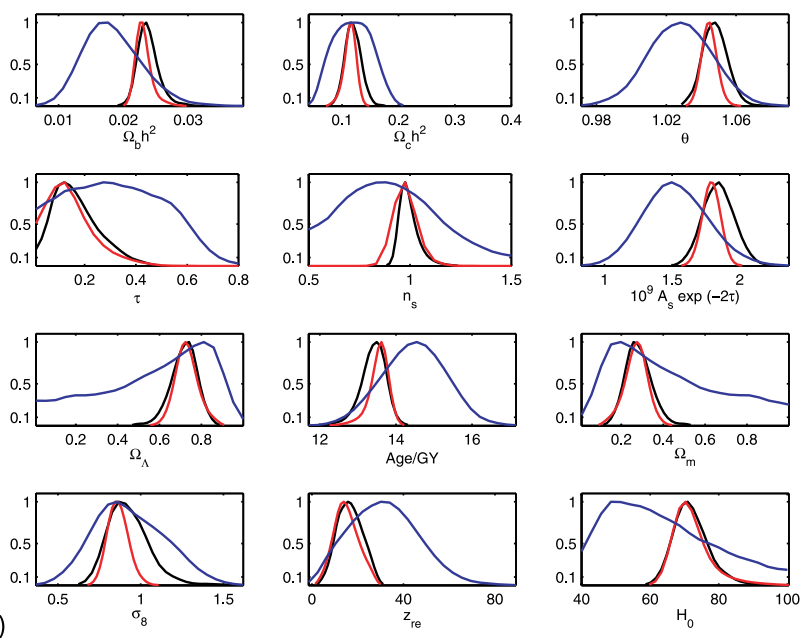

FIG. 7.-One-dimensional likelihoods for the cosmological parameter indicated, marginalized over the other parameters. (a) $\mathrm{CBI}$ EE data (red) and CBI+DASI+B03 $\mathrm{EE}+\mathrm{TE}$ data (blue). The black line is the prior, calculated using a parameter run with

complete mapping of the full likelihood surfaces without using the compressed bandpowers. The one-band model $\mathcal{C}_{l}^{X}=q_{s} \mathcal{C}_{l}^{X(s)}$, where $\mathcal{C}_{l}^{X(s)}$ is the fiducial adiabatic model, yields for the CBI EE data a value of $q_{s}=1.02 \pm 0.14(68 \%)$ and a $11.7 \sigma$ detection relative to $q_{s}=0$; the $\mathrm{CBI} \mathrm{EE}+\mathrm{TE}$ data give a $12.4 \sigma$ detection. These can be compared with the $6.3 \sigma$ DASI EE detection reported in Leitch et al. (2005) and the $8.9 \sigma$ CBI EE detection reported in Readhead et al. (2004b; with no polarization point sources projected out; $7.0 \sigma$ with $20 \%$ removal). Alone, the new CBI TE data give $q_{s}=1.02 \pm 0.24$ and a $4.2 \sigma$ detection relative to $q_{s}=0$. The CBI TT data yield $q_{s}=1.12 \pm 0.05$, which is a $95 \sigma$ significance detection versus $q_{s}=0$.

To further complement the MCMC determinations, we consider the two-parameter template model ${ }^{14} \mathcal{C}_{l}^{X}=t q_{s} \mathcal{C}_{l\left(\theta_{0} / \theta\right)}^{X(s)}$, evalu-

\footnotetext{
13 In Readhead et al. (2004b), we also described a two-parameter "sliding comb" test of the phase relationship between TT and EE. This involved an underlying smooth $\mathcal{C}_{l}^{X(s)}$ with a sinusoidal pattern characterized by an angular phase shift $\phi$ designed to give the fiducial model forecast for $\mathrm{EE}$ when $\phi=0$. The best-fit CBI EE phase was $21^{\circ} \pm 40^{\circ}$ with amplitude $q_{s}=1.07 \pm 0.21$; the new data give $13^{\circ} \pm 36^{\circ}$ and $1.05 \pm 0.13$.
}

(a)

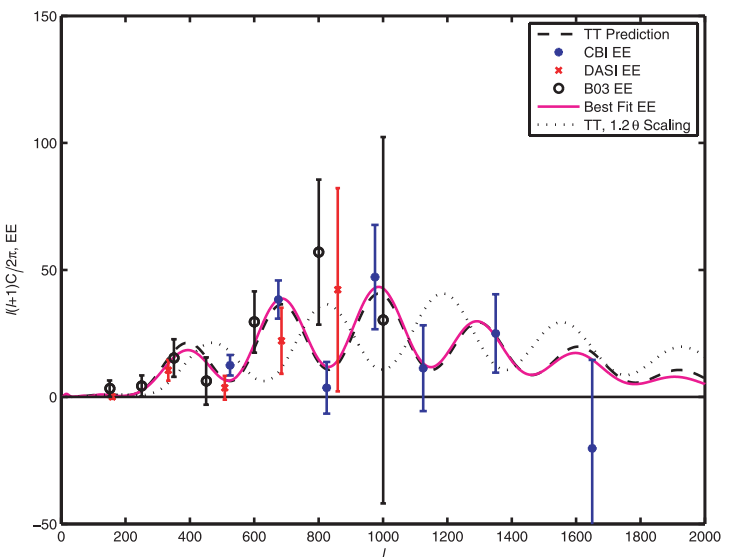

(b)

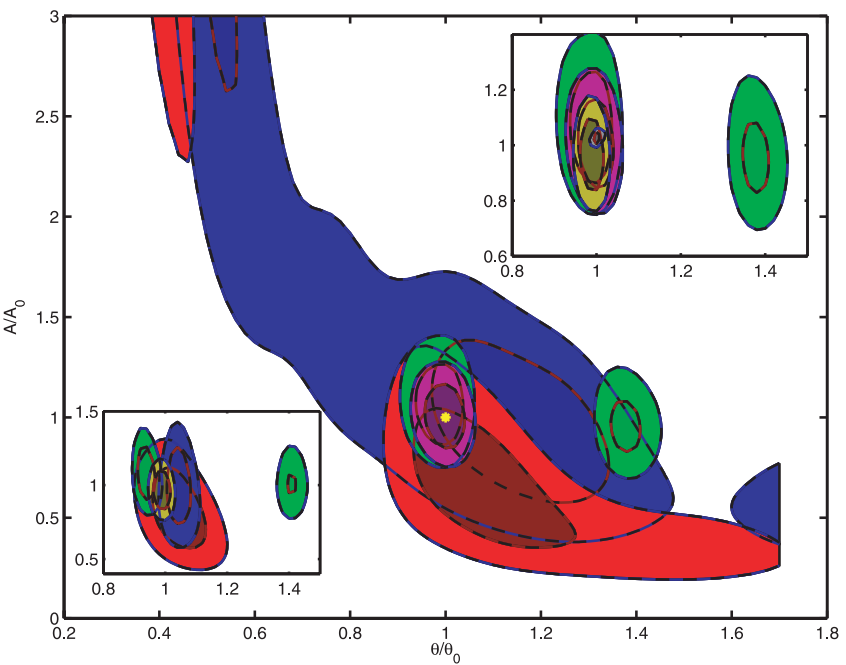

FIG. 8.- (a) Polarization data from CBI, DASI, and B03, along with the fiducial EE prediction (black dashed line), the same model shifted by $20 \%$ in $\theta$ (black dotted line), and the best-fit CBI+DASI+B03 EE cosmology (magenta line). The polarization data pick out the same sound-crossing angular scale as the TT data do, with $\theta / \theta_{0}=0.986 \pm 0.017$ when marginalized over the other five cosmological parameters. (b) Contours at 1 and $2 \sigma$, derived from CBI EE (green), DASI EE (red), B03 EE (blue) and CBI+DASI+B03 EE data (magenta) for the two-parameter $q_{s}$ and $\theta / \theta_{0}$ template model. Marginalization yields $\theta / \theta_{0}=$ $0.993 \pm 0.027$ and $q_{s}=1.00 \pm 0.11$. The yellow star marks the expected result from the fiducial model. The lower inset shows the same with EE+TE instead of EE only. The upper inset shows the sequence CBI EE ( green), CBI+DASI+B03 $\mathrm{EE}$ (magenta), and $\mathrm{CBI}+\mathrm{DASI}+\mathrm{B} 03 \mathrm{EE}+\mathrm{TE}$ (brown). Marginalization yields $\theta / \theta_{0}=0.988 \pm 0.018$ and $q_{s}=0.97 \pm 0.09$. The small gray contours denote $\mathrm{CBI}+\mathrm{B} 03 \mathrm{TT}$.

ated on a grid in $\left(q_{s}, \theta / \theta_{0}\right)$. The other cosmic parameters are fixed at the fiducial model values. We restrict $\theta / \theta_{0}$ to lie between 0.3 and 1.7 , the range of our grid. Figure $8 a$ shows how the EE peak/dip pattern shifts for the polarization. The $\theta / \theta_{0}-q_{s}$ likelihood contours in Figure $8 b$ show that for each of the EE polarization data sets there is a multimodal probability structure. For example, for CBI, apart from the $\theta / \theta_{0} \approx 1$ solution, there is another with the third polarization peak shifted and scaled to fit the second peak of the fiducial model. There is a strong probability minimum in between the two. This multiple solution disappears when DASI and B03 are combined with CBI, yielding the well-determined values of $\theta / \theta_{0}=0.988 \pm 0.018$ and $q_{s}=0.97 \pm 0.09$ for EE $+\mathrm{TE}$. These are in good agreement with the $0.986 \pm 0.017$ and $q_{s}=0.86 \pm$ 0.14 MCMC numbers determined by marginalizing over the other 

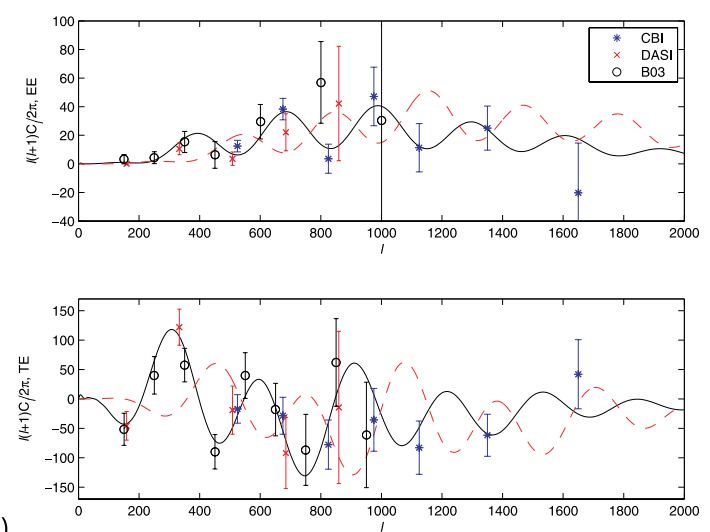

(a)

(b)

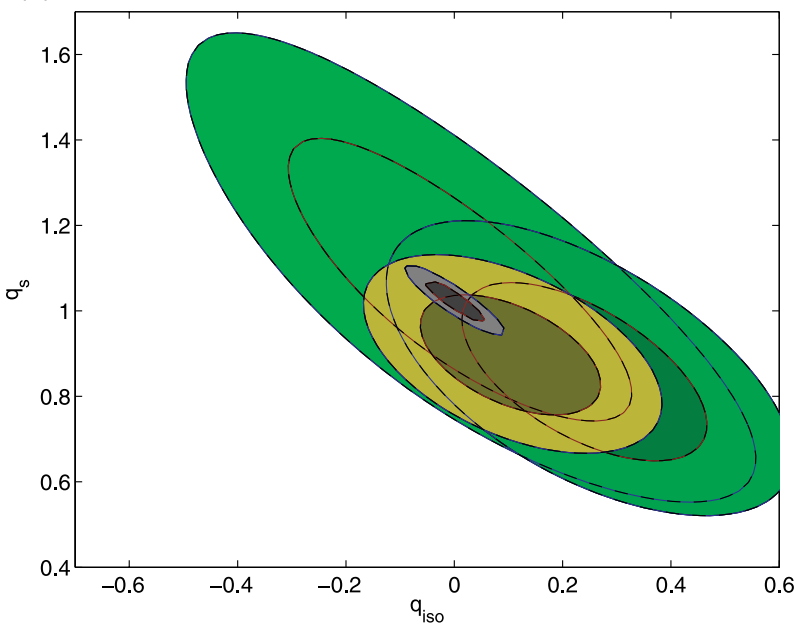

FIG. 9.-(a) $\mathcal{C}_{l}^{\mathrm{EE}(\text { iso) }}$ and $\mathcal{C}_{l}^{\mathrm{TE}(\text { iso) }}$ power spectra for the $n_{\text {iso }}=3$ white-noise isocurvature model (red dashed line) compared with $\mathcal{C}_{l}^{\mathrm{EE}(s)}$ and $\mathcal{C}_{l}^{\mathrm{TE}(s)}$ for the bestfit adiabatic fiducial model (black line). These are the template spectra used for the two-parameter $q_{s}-q_{\text {iso }}$ test. The quantity $q_{\text {iso }}$ is normalized to give the fraction of the expected EE power that is observed in the data over the range $400 \leq$ $l \leq 1200$. The CBI (blue asterisks), DASI (red crosses), and B03 (black circles) $\mathrm{EE}$ and TE data are also shown. (b) Likelihood surface for the two-parameter $q_{s}-q_{\text {iso }}$ model, for the CBI EE (light green), $\mathrm{CBI} \mathrm{EE}+\mathrm{TE}$ (dark green), $\mathrm{CBI}+\mathrm{DASI}+$ B03 EE+TE (brown), and CBI+B03 TT (gray) data. The data strongly prefer the adiabatic over the isocurvature spectrum. Marginalization over the twodimensional distributions yields the following: for $\mathrm{CBI} \mathrm{EE}, q_{s}=1.05 \pm 0.22$ and $q_{\text {iso }}=-0.01 \pm 0.21$; for CBI TE, $q_{s}=0.81 \pm 0.24$ and $q_{\text {iso }}=0.49 \pm 0.26$; and for CBI+DASI+B03 EE+TE data, $q_{s}=0.90 \pm 0.10$ and $q_{\text {iso }}=0.10 \pm 0.11$. The polarization data are consistent with a single-component adiabatic inflation model.

five cosmic parameters. The multimodal aspect is strongly suppressed in MCMC because of the extremely weak $h$ prior we impose, but correlations among parameters lead to the larger errors in $q_{s}$. For EE alone, the template grid gives $\theta / \theta_{0}=0.993 \pm$ 0.027 and $q_{s}=1.00 \pm 0.11$, and the marginalized MCMC gives $\theta / \theta_{0}=1.003 \pm 0.027$ and $q_{s}=1.07 \pm 0.30$.

Figure $8 a$ shows the best-fit EE power spectrum. It looks remarkably like the TT fiducial model forecast.

\subsection{Adding a Subdominant Isocurvature Mode}

Isocurvature modes that could lead to a measurable signal in the CMB may arise in multiple scalar field models during inflation, or they can be generated after inflation has ended. Necessary ingredients to impact CMB and large-scale structure (LSS) observations include association with a component of significant mass-energy, such as baryons, cold dark matter, or, possibly, massive neutrinos (hot dark matter), and sufficiently large primordial fluctuations in the entropy per baryon, the entropy per CDM particle, or the entropy per neutrino. A concrete CDM realization is the axion. For cosmic defect-induced isocurvature perturbations, which could also arise near the end of or after inflation, the mass-energy is in the defects.

Isocurvature perturbations from quantum zero-point fluctuations in inflation have a well-defined pattern (Efstathiou \& Bond 1986; Bond \& Efstathiou 1987) in TT, EE, and TE, with no BB predicted (except through lensing). The peaks and dips are predicted to be out of phase with those from adiabatic modes, as shown in Figure 9. (For defects it is difficult to get any peaks and troughs at all.) Further, there is a large "isocurvature effect" predicted at lower $l$ relative to that at high $l$, where the peaks are.

A pure isocurvature mode does not fit the current TT data unless the primordial isocurvature power spectrum $\mathcal{P}_{\text {iso }}(k)$ is designed to mimic the observed $\mathcal{C}_{l}^{\mathrm{TT}}$ pattern with its own peak/ dip structure and overall $k$-dependent blue tilt. Although highly baroque in terms of inflation models, such a radically broken scale invariance is possible for isocurvature perturbations, just as it is for the adiabatic case. Polarization (and LSS) data help by breaking such severe degeneracies with the cosmic parameters.

A detailed analysis of a general set of isocurvature initial conditions for four cosmological fluids using CMB and LSS data has been undertaken in Moodley et al. (2004). If one includes all allowed isocurvature and adiabatic perturbations and correlations between them, the current CMB and LSS data still allow a substantial amount of isocurvature perturbations. However, simpler and more realistic models that only include an isocurvature perturbation in one fluid are more strongly constrained.

Here we assume Gaussian-distributed CDM isocurvature perturbations and add two extra parameters beyond our base adiabatic six: two amplitude ratios, $R_{i} \equiv \mathcal{P}_{\text {iso }}\left(k_{i}\right) / \mathcal{P}_{s}\left(k_{i}\right)$, at two pivot wavenumbers $k_{i}$, one at small scale, $k_{2}=k_{n}=0.05 \mathrm{Mpc}^{-1}$, and one at large scale, $k_{1}=0.005 \mathrm{Mpc}^{-1}$. A (constant) primordial spectral index defined by $\mathcal{P}_{\text {iso }}(k) \propto k^{n_{\text {iso }}}$ follows: $n_{\text {iso }}=n_{s}-1+$ $\ln \left(R_{2} / R_{1}\right) / \ln \left(k_{2} / k_{1}\right)$.

We find that for neither $R_{i}$ parameter is there evidence for an isocurvature detection, in agreement with MacTavish et al. (2006), who used the same $R_{1}-R_{2}$ parameterization. We find, for the WMAP $1+\mathrm{CBI}+\mathrm{DASI}+\mathrm{B} 03 \mathrm{TT}+\mathrm{EE}+\mathrm{TE}$ data, $95 \%$ confidence upper limits of $R_{1}<0.26$ and $R_{2}<1.7$ on the higher wavenumber scales that CBI probes. This translates into steeper $n_{\text {iso }}$ values being more allowed than the $n_{\text {iso }} \approx 0$ nearly scale-invariant ones. The CBI EE+TE data only limit $R_{1}<18$ and $R_{2}<54$, whereas the $\mathrm{CBI}+\mathrm{DASI}+\mathrm{B} 03 \mathrm{EE}+\mathrm{TE}$ data give $R_{1}<9$ and $R_{2}<30$.

Inflation models more naturally produce nearly scale-invariant isocurvature spectra, with $n_{\text {iso }} \approx 0$, just as one often gets $n_{s} \approx 1$ for adiabatic perturbations. The tilts from theory are also more likely to be red $\left(n_{\text {iso }}<0\right)$ than blue $\left(n_{\text {iso }}>0\right)$. However, the data more strongly constrain red models than blue.

\subsection{Constraints on Interloper Isocurvature Peaks}

To focus attention on the high- $l$ polarization results, we now fix $n_{\text {iso }}$ to be the extremely blue value of 3 , the white noise "isocurvature" spectrum, with no spatial correlation. Large angular scales in $\mathcal{C}_{l}^{X \text { (iso) }}$ are highly suppressed, and the isocurvature peaks and troughs emerge looking somewhat like an $l$-shifted version of the adiabatic spectrum, as shown in Figure 9. (The $n_{\text {iso }}=2$ case, which looks even more like a shifted version of the fiducial model, gives similar results to those given here. See MacTavish et al. [2006] for a treatment of both the $n_{\text {iso }}=2$ and 3 cases.) Although $\mathcal{P}_{\text {iso }}(k)$ is so steep for such blue spectra that it must be regulated 
by a cutoff at high $k \gg k_{2}, \mathcal{C}_{l}^{X \text { (iso) }}$ has a larger natural damping scale, so we do not need to add another parameter.

The two-parameter template model, $\mathcal{C}_{l}^{X}=q_{s} \mathcal{C}_{l}^{X(s)}+q_{\text {iso }} \mathcal{C}_{l}^{X \text { (iso) }}$, therefore tests at what level an interloper set of isocurvature peaks would be allowed by the CMB data, which, as we have seen in the $\theta / \theta_{0}$ test, prefer the adiabatic peak positions. We normalize $q_{\text {iso }}$ so that $q_{\text {iso }}=1$ corresponds to the same power in $\mathcal{C}_{l}^{\mathrm{EE}(\text { iso) }}$ as in $\mathcal{C}_{l}^{\mathrm{EE}(s)}$ over a band from $l=400$ to 1200 . We find $q_{\text {iso }} \approx R_{2} q_{s} / 80$. Figure $9 b$ shows a strong preference for the pure adiabatic mode and no isocurvature detection, with $q_{\text {iso }}=-0.01 \pm 0.21$ for the CBI EE data, $0.24 \pm 0.15$ for the CBI EE+TE data, and $0.10 \pm 0.11$ for all of the polarization data.

We also let the full seven cosmological parameters vary, using CosmoMC to evaluate the probability distribution for $R_{2}$. This is a different exercise than the two-parameter case: to match the data, the other parameters are adjusted by CosmoMC to make the isocurvature troughs and peaks interfere with the adiabatic peaks and troughs, respectively, to mimic no interloping at all. For the CBI EE+TE data we get $R_{2}<76$, whereas for the $\mathrm{CBI}+$ $\mathrm{DASI}+\mathrm{B} 03 \mathrm{EE}+\mathrm{TE}$ data we get $R_{2}<44$. For the WMAP $1+\mathrm{CBI}+$ $\mathrm{DASI}+\mathrm{B} 03 \mathrm{TT}+\mathrm{EE}+\mathrm{TE}$ data, we get $R_{2}<3.0$. We note that $\mathcal{C}_{B}^{\mathrm{TT}(\text { iso })} / \mathcal{C}_{B}^{\mathrm{TT}(s)} \sim R_{2} / 80$, the same as for the EE ratio. Thus, the upper limits correspond to an allowed CMB contamination of this subdominant component of only $\sim 3 \%$. For the EE+TE data, $q_{\text {iso }}=0.16 \pm 0.21$ with an upper limit of $55 \%$, similar to the template value.

\section{CONCLUSIONS}

In this paper we present the results from $2.5 \mathrm{yr}$ of dedicated polarization-optimized measurements with the Cosmic Background Imager. From these data, we estimate the TT, TE, EE, and $\mathrm{BB} C \mathrm{CMB}$ angular power spectra. The EE power spectrum gives us a $11.7 \sigma$ detection of polarization, the strongest thus far, while TE is measured at $4.2 \sigma$ versus zero. The BB spectrum gives a $95 \%$ confidence upper limit of $3.8 \mu \mathrm{K}^{2}$.

We introduce a novel method for the reconstruction of $l$-space maps of $\tilde{E}$ and $\tilde{B}$. Images of the $E$ and $B$ fields on the sky are formed by a Fourier transform of the $l$-space maps; this is a new way of representing CMB polarization and is complementary to the standard Stokes $Q$ and $U$ images shown previously in Readhead et al. (2004b). The $E$-mode detection and the lack of one in $B$ is evident in both the raw maps and the reconstructed Wiener-filtered images of the $20^{\mathrm{h}}$ strip and is also evident in the square mosaic fields. We have also verified that signal-map fluctuations, shown in Figures $3 c$ and $3 d$, about the mean signal in Figure $3 b$ do not obscure this clear detection: the $20^{\mathrm{h}}$ strip is indeed dominated by the CMB polarization signal. The signal maps of the total intensity, an example of which is shown in Figure 6 for the $02^{\mathrm{h}}$ mosaic, also show very strong detections.
An analysis of a six-dimensional space of cosmological parameters shows that the patterns and amplitudes in the EE, TE, and $\mathrm{BB}$ data are entirely consistent with the basic inflation-based model predictions from TT, a result considerably strengthened by the new CBI EE+TE data. The combined CBI+DASI+B03 $\mathrm{EE}+\mathrm{TE}$ data further sharpen this conclusion. This is particularly evident in Figure 8, which shows that $\theta / \theta_{0}$, parameterizing the angular scale associated with sound crossing at decoupling and hence the peak-dip pattern, is pinned down to the value that we obtain from TT alone.

We finally explore a restricted physically motivated class of models with combined, but uncorrelated, adiabatic and isocurvature perturbations. We find that there is effectively no evidence for an isocurvature mode in the data. Furthermore, the data rule out a possible family of interloper peaks that would be out of phase with the standard flat adiabatic predictions. This strengthens our claim that cosmological models with an additional isocurvature mode are disfavored by the current polarization data.

We are indebted to the DASI team of the Kavli Institute for Cosmological Physics at the University of Chicago, led by John Carlstrom, and especially to John Kovac, who designed the achromatic polarizers. We thank the Kavli Operating Institute, Barbara and Stanley Rawn, Jr., Maxine and Ronald Linde, Cecil and Sally Drinkward, Rochus Vogt, and the Provost, President, and PMA Division Chairman of the California Institute of Technology for their generous support. We gratefully acknowledge support from the Canadian Institute for Advanced Research, the Canadian Space Agency, and NSERC at CITA. This research used the MacKenzie cluster at CITA, funded by the Canada Foundation for Innovation. This work was supported by the National Science Foundation under grants AST 94-13935, AST 98-02989, AST 00-98734, and AST 02-06416. R. B. was supported partially by CONICYT. L. B. and J. M. acknowledge support from the Chilean Center for Astrophysics FONDAP grant 15010003, and S. T. acknowledges support from grant Milenio ICM P02-049. This work was supported by the Leverhulme Trust and PPARC at Oxford. A. C. T. acknowledges support from the Royal Society. We thank CONICYT for granting permission to operate within the Chanjnantor Scientific Preserve in Chile, and the National Radio Astronomy Observatory (NRAO) Central Development Lab for developing the HEMT amplifiers used in this project and assisting with production. The National Radio Astronomy Observatory is a facility of the National Science Foundation operated under cooperative agreement by Associated Universities, Inc. We thank Jo Dunkley, Carrie MacTavish, and Mike Nolta for helpful comments. We thank Wilson Araya and José Cortes for their dedicated work in operating the CBI.
Barkats, D., et al. 2005, ApJ, 619, L127

Bennett, C., et al. 2003, ApJS, 148, 97

Bond, J. R., Contaldi, C. R., \& Pogosyan, D. 2003, Philos. Trans. R. Soc. London A, 361, 2435

Bond, J. R., \& Efstathiou, G. 1984, ApJ, 285, L45 1987, MNRAS, 226, 655

Bond, J. R., Jaffe, A. H., \& Knox, L. E. 2000, ApJ, 533, 19

Bunn, E. F., Zaldarriaga, M., Tegmark, M., \& de Oliveira-Costa, A. 2003, Phys. Rev. D, 67, 023501

Cartwright, J. K., Pearson, T. J., Readhead, A. C. S., Shepherd, M. C., Sievers, J. L., \& Taylor, G. B. 2005, ApJ, 623, 11

Efstathiou, G., \& Bond, J. R. 1986, MNRAS, 218, 103

Högbom, J. A. 1974, A\&AS, 15, 417

\section{REFERENCES}

Jones, W., et al. 2006, ApJ, 647, 823

Kogut, A., et al. 2003, ApJS, 148, 161

Kovac, J., Leitch, E. M., Pryke, C., Carlstrom, J. E., Halverson, N. W., \& Holzapfel, W. L. 2002, Nature, 420, 772

Leitch, E. M., Kovac, J. M., Halverson, N. W., Carlstrom, J. E., Pryke, C., \& Smith, M. W. E. 2005, ApJ, 624, 10

Lewis, A., \& Bridle, S. 2002, Phys. Rev. D, 66, 103511

Lewis, A., Challinor, A., \& Turok, N. 2002, Phys. Rev. D, 65, 023505

MacTavish, C., et al. 2006, ApJ, 647, 799

Masi, S., et al. 2006, A\&A, 458, 687

Montroy, T., et al. 2006, ApJ, 647, 813

Moodley, K., Buchler, M., Dunkley, J., Ferreira, P. G., \& Skordis, C. 2004, Phys. Rev. D, 70, 103520 
Myers, S. T., et al. 2003, ApJ, 591, 575

Padin, S., et al. 2002, PASP, 114, 83

Pearson, T. J., et al. 2003, ApJ, 591, 556

Piacentini, F., et al. 2006, ApJ, 647, 833

Readhead, A. C. S., et al. 2004a, ApJ, 609, 498 2004b, Science, 306, 836
Sievers, J. L., et al. 2003, ApJ, 591, 599

Spergel, D. N., et al. 2003, ApJS, 148, 175

Tucci, M., Martinez-Gonzalez, E., Toffolatti, L., Gonzalez-Nuevo, J., \& De Zotti, G. 2004, MNRAS, 349, 1267

Verde, L., et al. 2003, ApJS, 148, 195 